\title{
Cartilage intermediate layer protein is regulated by mechanical stress and affects extracellular matrix synthesis
}

\author{
JINYUE HE, CHENCHENG FENG, JING SUN, KANG LU, TONGWEI CHU, YUE ZHOU and YONG PAN
}

Department of Orthopaedics, Xinqiao Hospital, The Third Military Medical University, Chongqing 400037, P.R. China

Received September 21, 2016; Accepted July 17, 2017

DOI: $10.3892 / \mathrm{mmr} .2018 .8588$

\begin{abstract}
Lumbar disc disease (LDD) is common in aged populations, and it is primarily caused by intervertebral disc degeneration (IDD). Cartilage intermediate layer protein (CILP), which is specifically expressed in intervertebral discs (IVDs), is suspected to be associated with IDD. However, it remains unclear whether CILP contributes to IDD in humans. Furthermore, the regulation of CILP in human IVDs is poorly understood, especially by mechanical stimuli, which are regarded as primary factors promoting IDD. To address these issues, the present study collected nucleus pulposus (NP) cells from patients undergoing lumbar spinal surgery for degenerative disc disease (DDD). Subsequently, CILP expression was measured in human NP cells in response to mechanical stimuli, including cyclic compressive stress and cyclic tensile strain (CTS), by reverse transcription-quantitative polymerase chain reaction and western blotting. Aggrecan and collagen II, which are the main components of the extracellular matrix (ECM) and traditional degenerative markers for IDD, were detected following the treatment with CILP small interfering (si)RNA or recombinant human CILP (rhCILP) at various concentrations to determine whether CILP contributes to IDD by negatively regulating expression of the ECM. The results revealed that CILP expression in loaded NP cells was significantly increased compared with that in non-loaded cells under compressive loading, and that it was markedly decreased in cells under tensile loading, in contrast with the expression of aggrecan and collagen II in response to the same stimuli. Furthermore, CILP siRNA effectively inhibited CILP expression and significantly increased the expression of aggrecan and collagen II. In addition, treatment of NP cells with a high concentration of rhCILP resulted in significantly decreased expression of aggrecan and
\end{abstract}

Correspondence to: Professor Yong Pan, Department of Orthopaedics, Xinqiao Hospital, The Third Military Medical University, 183 Xinqiao Main Street, Shapingba, Chongqing 400037, P.R. China

E-mail: bravepan@163.com

Key words: cartilage intermediate layer protein, nucleus pulposus cell, extracellular matrix, mechanical stress, intervertebral disc degeneration collagen II. In conclusion, these results demonstrated for the first time, to the best of our knowledge, that in human NP cells, CILP is regulated by mechanical stress and that its expression affects ECM synthesis. Therefore, CILP represents a promising therapeutic target for preventing loss of the matrix during IDD as a novel treatment strategy.

\section{Introduction}

Lower back pain is a common disorder that has attracted much attention due to its effects on human health. Up to $80 \%$ of individuals worldwide may experience lower back pain at some point in their lives, of whom a large proportion receive surgery, resulting in heavy burdens on families and societies. Intervertebral disc degeneration (IDD) is regarded as a primary contributor to the pathological process of lower back pain (1). As human IDD is a complex and multifactorial process with contributions from genes, mechanical stresses, cellular senescence and decreased nutrition $(1,2)$, more studies are required to further investigate the mechanism of IDD and to identify specific factors that may serve important roles in this condition.

Cartilage intermediate layer protein (CILP) is specifically expressed in intervertebral discs (IVDs) (3), and its expression is increased with ageing and disc degeneration (4,5). Previous studies have identified a genetic correlation of CILP with IDD among the upregulated genes in IDD (6-9), and a functional single nucleotide polymorphism (SNP) of the CILP gene has been demonstrated to increase the direct interaction of CILP with transforming growth factor- $\beta$ (TGF- $\beta$ ) and to prevent TGF- $\beta$ from activating the transcription of cartilage matrix genes in rabbit nucleus pulposus (NP) cells (3). In addition, increased CILP expression via $\mathrm{N}$-terminal domain-mediated inhibitory effects on chondrocyte insulin-like growth factor (IGF)-1 responsiveness has been demonstrated to impair chondrocyte growth and matrix repair and to indirectly promote polymeric precipitation inhibitor supersaturation in bovine ageing and osteoarthritis cartilage (10). Furthermore, CILP overexpression in transgenic mice has been reported to lead to a significantly decreased signal intensity of IVDs on magnetic resonance imaging, representing an early sign of degeneration $(11,12)$. These studies have suggested that CILP may not only function as a marker for but also have involvement in the pathogenesis of IDD.

The development of IDD in humans is a complex process that involves cell autophagy, cellular senescence, invasion of 
inflammatory mediators, up-regulation of catabolic enzymes, such as matrix metalloproteinases (MMPs) and a disintegrin and metalloproteinase with a thrombospondin motifs (ADAMTs), and downregulation of matrix proteins, such as aggrecan and collagen II $(1,2)$. Amongst the many events that occur in IDD, the disruption of homeostasis of the extracellular matrix (ECM) is regarded as one of the most important (2). Homeostasis of the ECM is important to maintain the normal structure and function of IVDs by allowing for interactions between resident cells and the extracellular environment (13). Once IDD is initiated, this homeostasis gradually breaks as an imbalance develops between the rates of production and breakdown of matrix components at early stages, leading to a series of events including alterations in the $\mathrm{pH}$ and osmotic pressure, thereby creating a harsh extracellular environment for resident cells in NP tissues. Furthermore, the ECM under healthy conditions is able to retain enough hydration to withstand and absorb the mechanical loading experienced in daily life (14). However, in IDD, synthesis of ECM components, including aggrecan and collagen II, which constitute the majority of the ECM, is decreased, leading to reduced hydration. Dehydration of the NP weakens its mechanical support of IVDs and exposes NP cells to excessive mechanical loading, which then stimulates increased catabolic responses in NP cells $(14,15)$. Hence, abnormal mechanical loading is one of the major factors promoting loss of the ECM in IDD, and it is an important mechanism through which mechanical loading contributes to IDD (14-16).

Although CILP has been suggested to interfere with the expression of matrix components, as mediated by several growth factors, in rabbit and mouse NP cells $(10,17)$, there is no direct evidence that CILP has a negative effect on homeostasis of the ECM in human IVDs. Furthermore, examination of alterations in CILP expression in human NP cells in response to different mechanical stimuli is required to obtain further insights into the regulation of CILP to support its potential role in IDD. To address these issues, the present study collected human NP cells from patients undergoing lumbar spinal surgery for degenerative disc disease (DDD) and detected the responses in NP cells, including the expression of CILP, aggrecan and collagen II, to mechanical stresses, including cyclic tensile strain (CTS) and cyclic compressive stress. In addition, the levels of aggrecan and collagen II, the main components of the ECM and traditional markers for IDD, were detected following treatment of NP cells with RNAi and rhCILP).

\section{Materials and methods}

Antibodies and reagents. Rabbit monoclonal anti-human CILP (cat. no. ab192881), collagen II (cat. no. ab188570) and polyclonal anti-human aggrecan (cat. no. ab36861) antibodies were purchased from Abcam (Cambridge, MA, USA). rhCILP was obtained from R\&D Systems (Minneapolis, MN, USA). Specific small interfering (si)RNA duplexes targeting human CILP and reduced-serum transfection medium were purchased from Santa Cruz Biotechnology, Inc. (Dallas, TX, USA), and the siRNA transfection reagent was purchased from Invitrogen; Thermo Fisher Scientific, Inc. (Waltham, MA, USA).

Ethics statement. Human IVDs were obtained with the approval of the Ethical Committee of Xinqiao Hospital (Chongqing,
China) and with informed consent, in accordance with the Helsinki Declaration, from individuals undergoing surgery for IDD.

Tissue collection and isolation and culturing of NP cells. Human IVD tissues were collected from 20 patients (Male: Female, 1:1) ranging in age from 30-60 years who were undergoing lumbar spinal surgery for DDD at the Department of Orthopaedics between January 2016 and May 2016, The Second Affiliated Hospital (Chongqing, China). The NP tissues were carefully minced and digested to obtain NP cells, as previously reported (18). Cells were cultured in Dulbecco's modified Eagle's medium (DMEM; Invitrogen; Thermo Fisher Scientific, Inc.) supplemented with penicillin $(50 \mathrm{U} / \mathrm{ml})$, streptomycin $(50 \mathrm{U} / \mathrm{ml})$, and $10 \%$ fetal bovine serum (FBS; Invitrogen; Thermo Fisher Scientific, Inc.). Subconfluent NP cells (passage number $<3$ ) were trypsinized (Gibco; Thermo Fisher Scientific, Inc.) and resuspended in complete medium to prepare for subsequent experiments.

Application of CTS. An equal amount of each cell suspension was transferred onto a silicone membrane of a BioFlex 6-well tension plate (Flexcell International, Dunn Labortechnik, Asbach, Germany) at a density of $5 \times 10^{5}$ cells/well and were allowed to adhere for $48 \mathrm{~h}$. The medium was replenished at $12 \mathrm{~h}$ before application of a CTS. A CTS of $10 \%$ at a $1.0 \mathrm{~Hz}$ frequency was delivered to the bases of the silicone membranes within the Bioflex culture plates using an FX-5000C ${ }^{\text {TM }}$ Flexcell system, and NP cells adhered to the membranes via computer-controlled negative pressure for different durations $(0,6,12,24$ and $48 \mathrm{~h})$. Cells without tensile loading (control group) were cultured in the same culture plates as the cells in the stimulation groups. Mechanically stimulated and unstimulated NP cells were incubated at $37^{\circ} \mathrm{C}$ with $5 \% \mathrm{CO}_{2}$ during stimulation.

Application of cyclic compressive stress. Cell suspensions at a concentration of $2 \times 10^{6} / \mathrm{ml}$ were prepared in tubes and were then mixed with carbonate buffer solution ( $\mathrm{pH} \mathrm{5.5),}$ maleimide-polymer (vinyl alcohol), water and PEG-Link according to the manufacturer's protocol (3D Life Dextran-PEG Hydrogel kit G90-1, Cellendes, Germany). The reaction was mixed via rapid vortexing for no more than $5 \mathrm{sec}$. Each cell suspension $(1 \mathrm{ml})$ was obtained using a $1,000-\mu 1$ pipette tip (Corning Incorporated, Corning, NY, USA) and a pipette (Eppendorf, Hamburg, Germany) prior to gel formation. The front end of the pipette tip was used to obtain a cross-section of $5 \mathrm{~mm}$ in diameter. The gel in the pipette tip was then extruded and sliced into cylinders with a height of $3 \mathrm{~mm}$ that were suitable for placement in the inner foam rings of Bioflex 6-well compression plates (Flexcell International, Dunn Labortechnik, Asbach, Germany). These cylinders were placed into the Bioflex plates according to the manufacturer's protocol. DMEM-F12 containing $10 \%$ serum was added to each plate to immerse the cylinders in medium. A compressive loading of 1-2.5 $\mathrm{MPa}$ at a $1.0 \mathrm{~Hz}$ frequency was delivered to the bases of the silicone membranes within the Bioflex plates using an FX-5000C ${ }^{\text {TM }}$ Flexercell system and then to NP cells residing in the gel. The stimulation groups were subjected to stimuli for different periods of time $(6,12,24$ and $48 \mathrm{~h})$, and cells without a tensile load (control group) were cultured in the same culture plates as those 
in the other groups. Mechanically stimulated and unstimulated $\mathrm{NP}$ cells were incubated at $37^{\circ} \mathrm{C}$ and $5 \% \mathrm{CO}_{2}$ during stimulation.

Extraction of cells from the gel following compressive loading. The gels in the plates were transferred to sterile centrifuge tubes, and $100 \mu$ l glucanase each tube, provided in a 3D Life Dextran-PEG Hydrogel kit G90-1 (Cellendes GmbH, Reutlingen, Germany), was added to the tubes. Once the gels had dissolved, the suspended cells were harvested via centrifugation $(500 \mathrm{x} \mathrm{g}$, 6 min, room temperature).

Transfection with siRNA. Subconfluent NP cells (passage number $<4$ ) were equally divided into the siRNA group, the negative control group and the blank control group, and then were transferred to cell culture plates at a density of $5 \times 10^{5}$ cells/well. siRNA transfection was conducted using Lipofectamine iMax (Invitrogen; Thermo Fisher Scientific, Inc.) according to the manufacturer's protocol. The final siRNA (cat. no. sc-60384; Santa Cruz Biotechnology, Inc.) concentration was $100 \mathrm{nM}$. Cells in the control group were cultured in the same culture medium without siRNA. Negative control siRNA (cat. no. sc-37007; Santa Cruz Biotechnology, Inc.) was used to evaluate the off-target effects of RNAi and to verify the accuracy of gene-specific siRNA-dependent RNAi. Knockdown efficiency was detected at $48 \mathrm{~h}$ after transfection by reverse transcription-quantitative polymerase chain reaction (RT-qPCR) and western blotting.

Treatment with rhCILP at various concentrations. Subconfluent NP cells (passage number $<4$ ) were equally divided into the treatment groups and the control group and then were transferred to cell culture plates at a density of $5 \times 10^{5}$ cells/well. The cells were allowed to adhere for $24 \mathrm{~h}$ prior to treatment with rhCILP (10, 100 and $1,000 \mathrm{ng} / \mathrm{ml})$. An untreated group served as the control group. The cells were incubated at $37^{\circ} \mathrm{C}$ and $5 \% \mathrm{CO}_{2}$ for $72 \mathrm{~h}$ after treatment.

Extraction of total RNA and RT-qPCR. Total RNA was extracted using an RNeasy Mini Kit (Qiagen, Valencia, CA), and cDNA was reverse transcribed from $1 \mu \mathrm{g}$ total RNA using an Omniscript Reverse Transcription kit (Qiagen, Inc., Valencia, CA, USA) according to the manufacturer's protocol. RT-qPCR was performed with a ViiA7 Real-Time PCR system (Applied Biosystems; Thermo Fisher Scientific, Inc.) and a QuantiNova ${ }^{\mathrm{TM}}$ SYBR Green PCR kit (Qiagen, Inc.). Primers with the following sequences were used: Human GAPDH: F: 5'CCAGCAAGA GCACAAGAGGAAGAG3', R: 5'GGTCTACATGGCAACTGT GAGGAG3', human CILP: F: 5'AGCGGTGTACGGAAACTC G3', R: 5'ACGGCACTCCCCTTCTTGT3', human aggrecan: F: 5'TGAGGAGGGCTGGAACAAGTACC3', R: 5'GGAGGT GGTAATTGCAGGGAACA3' and human collagen II: F: 5'TTTCCCAGGTCAAGATGGTC3', R: 5'TCACCTGGTTTT CCACCTTC3'.

qPCR reaction was performed in triplicate. The $20 \mathrm{ml}$ reaction volume was applied. The reaction parameters were $95^{\circ} \mathrm{C}$ for $30 \mathrm{sec}$ followed by 40 cycles of $95^{\circ} \mathrm{C}$ for $5 \mathrm{sec}$ for template denaturation and $60^{\circ} \mathrm{C}$ for $34 \mathrm{sec}$ for annealing and extension. The results are presented as a $\mathrm{Cq}$ value, which is the cycle number at which the amplified product was first detected. The mean $\mathrm{Cq}$ value was obtained from three repeated experiments. The expression levels of the target genes were normalized to that of the endogenous control (GAPDH). The relative target mRNA expression levels were calculated using the $2^{-\Delta \Delta C q}$ method (19).

Western blot analysis. Total protein was extracted using radioimmunoprecipitation assay lysis buffer (Sigma-Aldrich; Merck KGaA, Darmstadt, Germany) with Halt ${ }^{\mathrm{TM}}$ Protease/Phosphatase Inhibitor (Thermo Fisher Scientific, Inc.) at $4^{\circ} \mathrm{C}$, with frequent agitation for $30 \mathrm{~min}$. Cell lysates were cleared of insoluble debris via centrifugation at $12,000 \mathrm{xg}$ for $30 \mathrm{~min}$ at $4^{\circ} \mathrm{C}$. The amount of total protein was determined by bicinchoninic acid protein assay (Pierce; Thermo Fisher Scientific, Inc.). Equal amounts of protein $(20 \mu \mathrm{g})$ were separated by $10 \%$ SDS-PAGE and transferred onto polyvinylidene difluoride membranes (Immobilon; EMD Millipore, Billerica, MA, USA), which were blocked with $5 \%$ milk for $1 \mathrm{~h}$ at $37^{\circ} \mathrm{C}$. The filters were incubated overnight at $4^{\circ} \mathrm{C}$ with primary rabbit antibodies diluted $1: 1,000$ and then with horseradish peroxidase-conjugated secondary antibodies (cat. no. ZB-2301; Origene Technologies, Inc., Beijing, China) diluted 1:2,500 for $1 \mathrm{~h}$ at room temperature. Bands were detected using an enhanced chemiluminescence system (EMD Millipore) and scanned using an ImageQuant LAS4000 imaging system (GE Healthcare, Chicago, IL, USA). The optical density (OD) of the bands was measured using ImageJ software (National Institutes of Health, Bethesda, MD, USA).

Statistical analysis. GraphPad Prism 6 (GraphPad Software, Inc., La Jolla, CA, USA) and SPSS version 22.0 statistical software (IBM Corp., Armonk, NY, USA) were used to analyse and display the data of this study. Each condition was performed in triplicate, and the mean was determined. All of the results are presented as the mean \pm standard deviation. Statistical comparisons were performed using a one-way analysis of variance, followed by Bonferroni's post hoc test. $\mathrm{P}<0.05$ was considered to indicate a statistically significant difference.

\section{Results}

Aggrecan and collagen II expression is significantly increased under tensile loading and decreased under compressive loading. Aggrecan and collagen II are the main components of the ECM, as well as traditional degenerative markers for IDD, and their expression levels decrease with ageing and degeneration. As presented in Fig. 1A, which demonstrated that there was a significant increase in mRNA level of aggrecans and collagen II subjected to tensile loading, with time (only up to $24 \mathrm{~h}$ in collagen II) was observed $(\mathrm{P}<0.05)$. The peak expression was observed in the $24 \mathrm{~h}$ group for collagen II, and in the 48-h group for aggrecan. With regard to the effect of compressive loading on matrix gene expression in human NP cells, the mRNA expression levels of aggrecan and collagen II were significantly decreased in all groups, and the lowest expression was observed in the 48-h group for collagen II, and in the 24-h group for aggrecan $(\mathrm{P}<0.05$; Fig. 1B). WB results demonstrated aggrecan expression under tensile loading was significantly increased $(\mathrm{P}<0.05$; Fig. $1 \mathrm{C})$ and decreased significantly under compression loading $(\mathrm{P}<0.05$; Fig. 1D), Similarly, the protein level of collagen II under tensile loading was significantly increased $(\mathrm{P}<0.05$; Fig. 1E) while decreased significantly under compression loading $(\mathrm{P}<0.05$; Fig. $1 \mathrm{~F})$. 

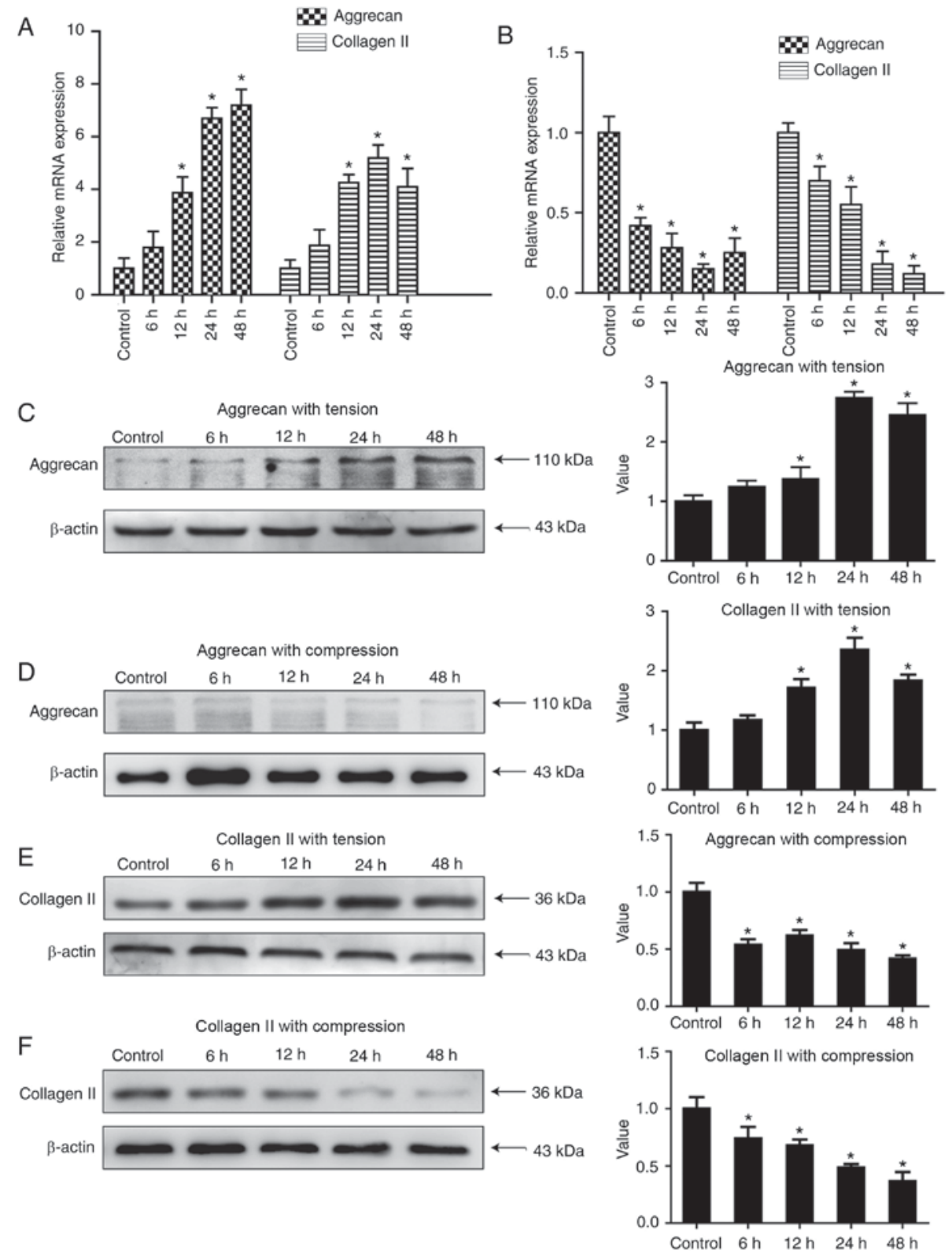

Figure 1. Expression of matrix genes in human nucleus pulposus cells under mechanical stress for various durations of stimulation. Reverse transcription-quantitative polymerase chain reaction analysis demonstrating the mRNA expression of aggrecan and collagen II under (A) tensile and (B) compressive loading. Representative western blot image for aggrecan under tensile (C) and collagen II under tensile is (E). Representative western blot image for aggrecan under compression is (D) and collagen II under tensile is (F). $\beta$-actin served as a loading control. Data are presented as the mean \pm standard deviation. "P $<0.05$ vs. control.

CILP expression is decreased under tensile loading and increased under compressive loading in contrast with that of aggrecan and collagen II. The regulations of mechanical loading on the synthesis of ECM by NP cells was revealed previously; therefore, the present study aimed to investigate whether mechanical loading can regulate CILP expression. The expression of CILP demonstrated an opposite trend as that of the matrix genes in tensile loading; they decreased in a time-dependent manner following compressive loading (Fig. 2A; $\mathrm{P}<0.05$ ), and increased in a time-dependent manner following tensile loading (Fig. 2B; $\mathrm{P}<0.05$ ). The protein expression levels demonstrated the same trend (Fig. $2 \mathrm{C}$ and $\mathrm{D}$, respectively; $\mathrm{P}<0.05$ ). Considering the ability of healthy NP tissues to buffer and transform mechanical loading to other mechanical loading including tension, it is reasonable to speculate the relatively low level of
CILP in healthy IVDs compared with degenerative IVDs is partly a result of tensile loading endured in daily life.

The present study further investigated the effect of compressive loading on CILP expression. A compressive loading was applied at 1.0-2.5 MPa at $1 \mathrm{~Hz}$, which is higher than that endured by NP cells in healthy IVDs in daily life. Consequently, compressive loading led to decreased aggrecan and collagen II expression, as well as markedly upregulated CILP expression, with peak expression detected in the 48-h group ( $\mathrm{P}<0.05$; Figs. 1 and 2$)$. The results above demonstrated that CILP expression is regulated by mechanical stimuli, and the regulation is dependent on the mechanical types. Furthermore, these results suggested it is the harsh mechanical loading in IVD that contributes to the abnormal high expression of CILP in degenerative IVDs. 
A

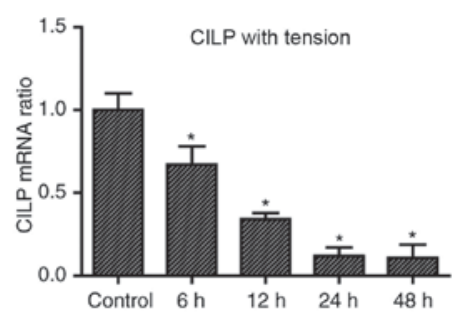

C

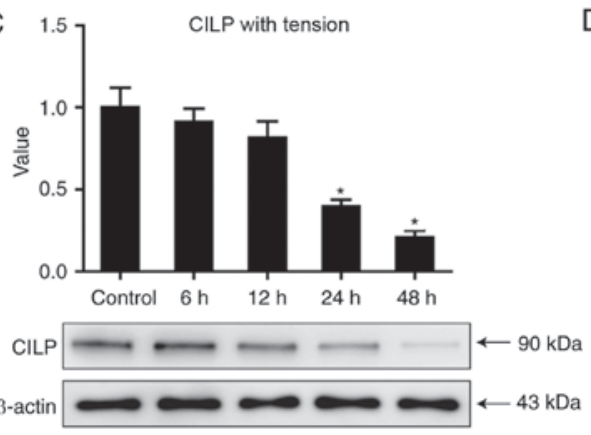

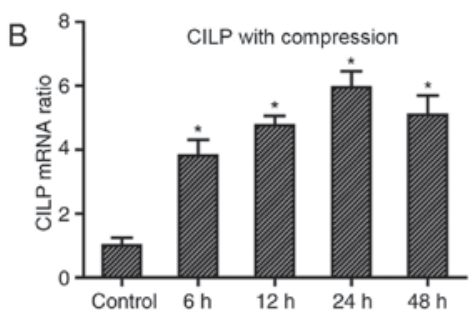

$\mathrm{D}$

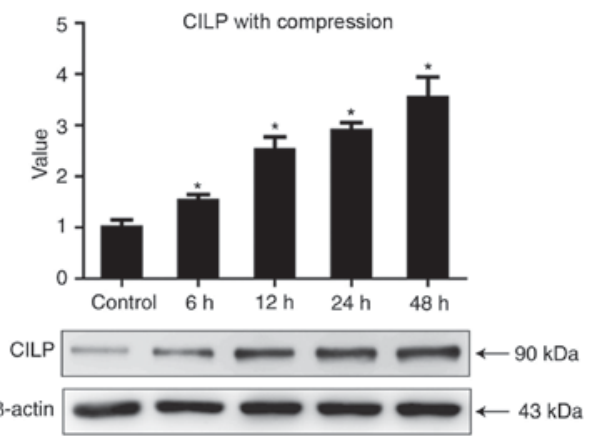

Figure 2. CILP expression in human nucleus pulposus cells under mechanical stress for various durations of stimulation. Reverse transcription-quantitative polymerase chain reaction analysis demonstrating the mRNA expression of CILP under (A) tensile and (B) compressive loading. Representative western blot images and quantification of CILP protein expression levels under (C) tensile and (D) compressive loading. $\beta$-actin served as a loading control. Data are presented as the mean \pm standard deviation. ${ }^{*} \mathrm{P}<0.05$ vs. control. CILP, cartilage intermediate layer protein.

Aggrecan and collagen II levels are increased following the silencing of CILP expression by RNAi. It is important to investigate whether excessive CILP expression by NP cells contributes to IDD. As previous studies have revealed the antagonism of CILP to TGF- $\beta$ in rabbit NP cells (5), The present study hypothesized that CILP may function as a negative factor in ECM synthesis in human NP cells. To prove the assumption, the present study used RNAi to inhibit CILP expression. The NP cells were transfected with $100 \mathrm{~nm}$ siRNA targeting the CILP gene. The results in Fig. 3 revealed that while CILP expression was effectively inhibited by siRNA $(\mathrm{P}<0.05)$, the expression of aggrecan and collagen II were markedly increased $(\mathrm{P}<0.05)$. Therefore, abnormal CILP expression may exert a negative effect on the synthesis of matrix genes in IDD.

Aggrecan and collagen II levels are increased following treatment of NP cells with high concentrations of rhCILP. To further verify the negative regulation of CILP on the synthesis of ECM, human NP cells were treated with various concentrations of rhCILP. The results in Fig. 4 demonstrated that expression of the matrix genes was markedly downregulated in the $1,000 \mathrm{ng} / \mathrm{ml}$ groups $(\mathrm{P}<0.05)$, illustrating that a high level of CILP expression inhibits the expression of matrix genes. No significant differences were detected in the 10 and $100 \mathrm{ng} / \mathrm{ml}$ groups compared with the control group $(\mathrm{P}>0.05)$, implying that the inhibition only has an effect when CILP expression is upregulated to a high degree. These results, along with the above findings, indicated that CILP expression is strongly associated with the homeostasis of ECM in IVDs, and a high CILP level in IDD may accelerate the degeneration by disrupting ECM homeostasis.

\section{Discussion}

The present study aimed to investigate the regulation of CILP by mechanical stress, and the association of CILP expression with the synthesis of aggrecan and collagen II, which are main components of the ECM that serve important roles in maintaining the normal structure and function of human IVDs. Consequently, to the best of our knowledge, the present study is the first to demonstrate that CILP expression is regulated by mechanical stress, and that its regulation varies with the type of mechanical stress. Furthermore, the results indicated that altered CILP expression has negative effects on the synthesis of aggrecan and collagen II.

Preliminary examinations of the correlation of CILP with IDD have been performed in previous studies. At the genetic level, CILP gene expression has been associated with IDD in Japanese and Finnish individuals (6-9). In rabbit NP cells, a functional SNP of the CILP gene has been reported to increase the direct interaction of CILP with TGF- $\beta$ and to interfere with the binding of TGF- $\beta 1$ to its receptors. This interference leads to suppression of the phosphorylation of mothers against decapentaplegic (Smad)2/3, which are key factors in the TGF- $\beta /$ Smad signalling pathway (3). Similarly, increased CILP expression exerts an inhibitory effect on chondrocyte IGF-1 responsiveness and impairs IGF-1-mediated chondrocyte growth and matrix repair in bovine chondrocytes (10). Thus, it seems that CILP may affect matrix synthesis via its interactions with several growth factors. However, there is no direct evidence of a role of CILP in matrix synthesis in human NP cells. To the best of our knowledge, these results are the first to demonstrate that in human NP cells, excessive CILP expression negatively regulates matrix synthesis. The results further imply that abnormal CILP expression may be one of the reasons for IDD, as suggested by previous studies. However, it remains unknown whether this negative control is a direct effect of interactions with NP cells or an indirect effect of interactions with several growth factors; further study is necessary to clarify this issue.

IVDs are load-bearing structures that undergo various deformations in response to the spine load in daily life, and 
A

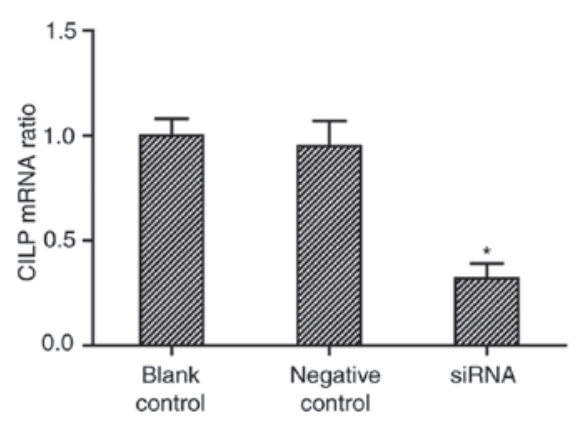

C
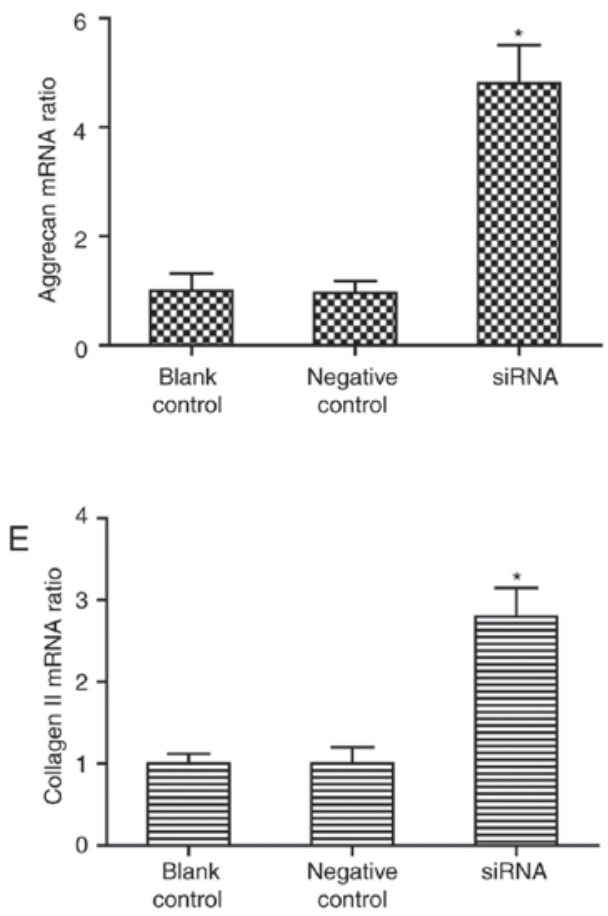

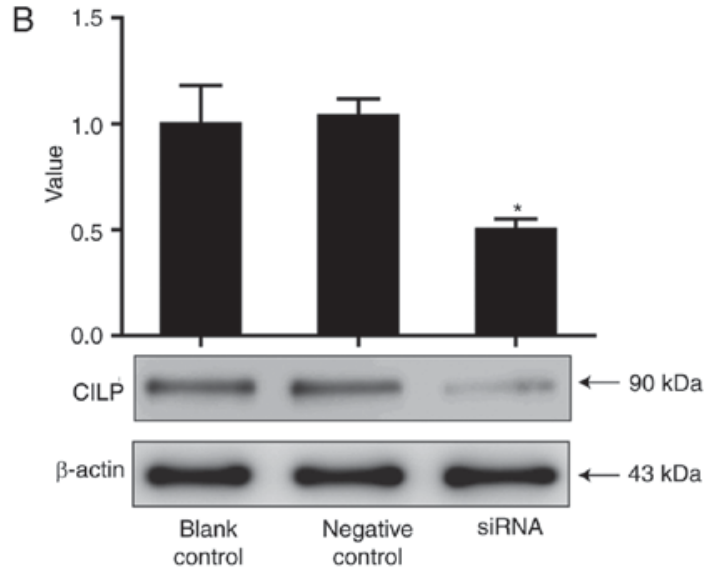

D

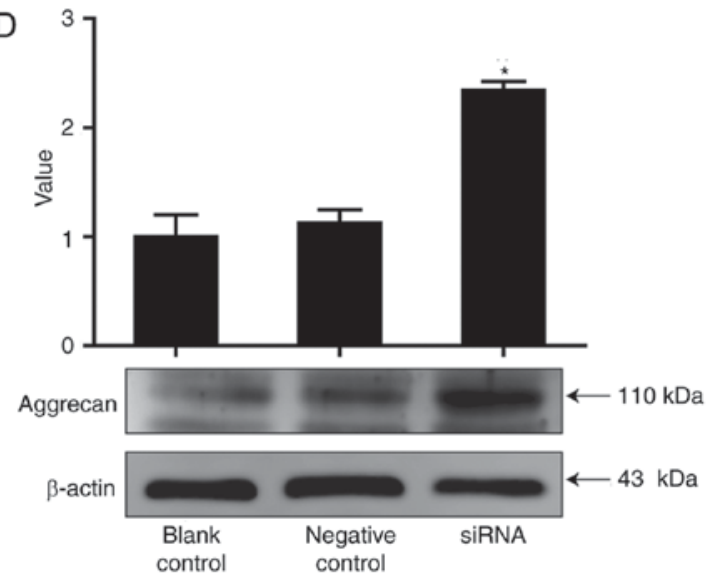

$\mathrm{F}$

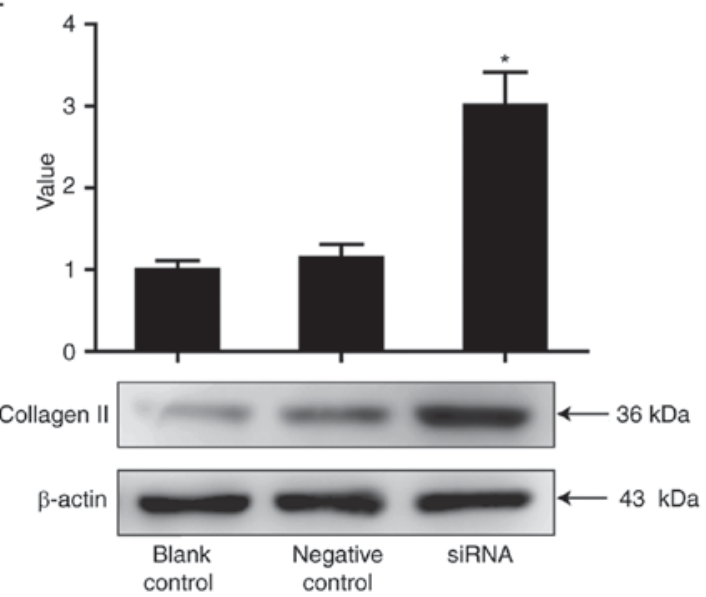

Figure 3. CILP expression is inhibited using siRNA. (A) RT-qPCR and (B) western blot analyses of mRNA and protein expression of CILP following knockdown of the CILP gene, respectively. (C) RT-qPCR and (D) western blot analyses of mRNA and protein expression of aggrecan following knockdown of the CILP gene, respectively. (E) RT-qPCR and (F) western blot analyses of mRNA and protein expression of collagen II following knockdown of the CILP gene, respectively. $\beta$-actin served as a loading control for western blotting. Data are presented as the mean \pm standard deviation. "P<0.05 vs. control. CILP, cartilage intermediate layer protein; RT-qPCR, reverse transcription-quantitative polymerase chain reaction; siRNA, small interfering RNA.

transfer the external mechanical load to resident cells in NP tissues $(15-17,20)$. These cells are susceptible to changes in the mechanical environment, and exposure to excessive stress promotes increased catabolic responses in NP cells, including upregulation of ADAMTs and MMPs and downregulation of aggrecan and collagen II (15-17,20-22). Therefore, homeostasis of the internal environment around NP cells relies on the dynamic balance between the external mechanical stimuli and the ability to buffer and absorb stress $(13,15,16,21,22)$. Once degeneration is initiated in IVDs, the aggrecan and collagen II levels in the ECM decrease during the early stages, resulting in reduced hydration (1), which weakens the ability of IVDs to buffer mechanical stress and causes NP cells to be exposed to more stressful stimuli, especially direct compressive loading, further promoting the catabolic responses in the $\operatorname{ECM}(1,13)$. In summary, mechanical stress, especially excessive stress, is a primary factor promoting IDD. Therefore, it is of significance to examine the regulation of CILP expression by mechanical stresses. Mechanical stimuli have various effects on metabolism in NP cells depending on the stimulus type, and it is impossible 

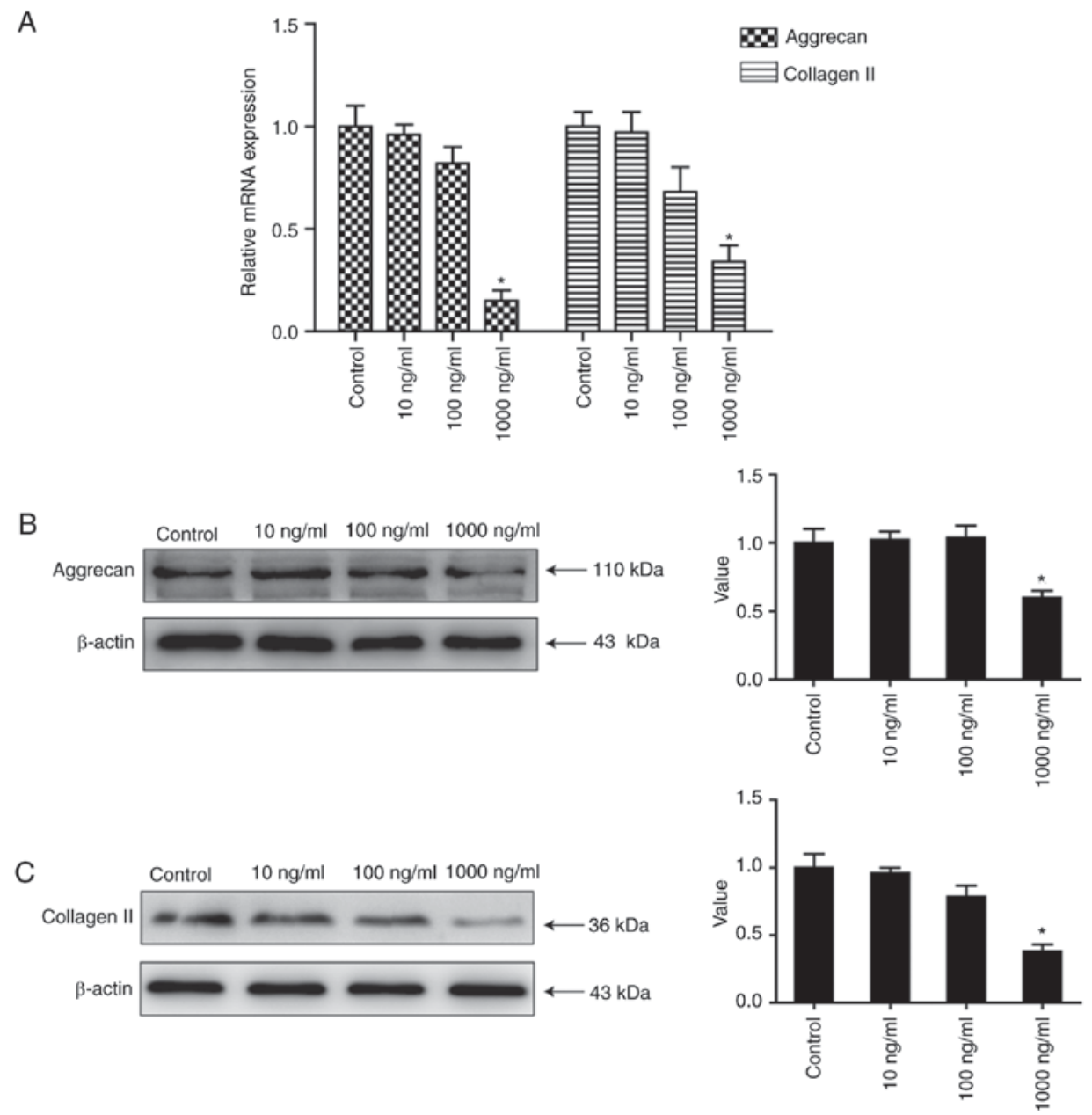

Figure 4. Expression of aggrecan and collagen II in nucleus pulposus cells treated with rhCILP at various concentrations (10, 100 and 1,000 ng/ml). (A) Reverse transcription-quantitative polymerase chain reaction analysis demonstrating the mRNA expression of aggrecan and collagen II in NP cells treated with rhCILP. Western blot analysis of (B) aggrecan and (C) collagen II protein expression levels following treatment with rhCILP. $\beta$-actin served as a loading control for western blotting. Data are presented as the mean \pm standard deviation of three independent experiments. ${ }^{*} \mathrm{P}<0.05$ vs. control. rhCILP, recombinant human cartilage intermediate layer protein.

to completely simulate the internal mechanical environment in vitro. The present study selected compression and tension, which are the main mechanical stimuli exerted on NP cells, to determine the response of CILP expression in NP cells to mechanical stress. Previous studies have demonstrated that the effect of compression on NP cells relies on the magnitude, frequency and duration. The compression experienced in daily life ranges from 0.1-1.3 MPa at a frequency $0.1-1.0 \mathrm{~Hz}(22)$, and compression of $>1 \mathrm{MPa}$ at a frequency of $1 \mathrm{~Hz}$ has been reported to increase catabolism in NP cells (15), while compression of $0.25 \mathrm{MPa}$ at a frequency of $0.1 \mathrm{~Hz}$ results in increased anabolism in these cells (21). Given that the NP cells used in the present study were derived from patients with degenerative IVDs compression of 1.0-2.5 MPa at a frequency of $1 \mathrm{~Hz}$ was selected to simulate the excessive stress faced by NP cells in these IVDs. With regard to tension, previous studies concerning tensile strain have demonstrated that $10 \%$ elongation evokes significant changes in cells, and the loading of cells with a 3-10\% strain primarily leads to anabolic responses in chondrocyte cells (23). To examine the effect of tensile stimuli on CILP expression, the present study selected $10 \%$ elongation at a frequency of $1 \mathrm{~Hz}$. The results revealed that under $10 \%$ tensile loading at $1 \mathrm{~Hz}$, mechanical stimuli that promoted the expression of matrix genes significantly decreased that of CILP. In addition, compressive loading of 1.0-2.5 MPa, an abnormal stimulus for human NP cells, resulted in the marked upregulation of CILP expression. These results demonstrated that CILP expression is regulated not only by ageing or growth factors $(5,10,17)$, but also by mechanical stress, and that the effect of mechanical regulation is dependent on the mechanical stress type. Given that the NP cells in degenerative IVDs are exposed to excessive compressive loading and insufficient tensile loading resulting from the weakened ability of IVDs to suffer and transform external mechanical stress. These results suggested that the high level of CILP in IDD is more likely to be a result of abnormal mechanical stress endured in daily life. Combined with the previous results concerning the effect of CILP on the synthesis of ECM, these experiments provided a novel explanation for the differential expression of CILP in IVDs with differing degrees of IDD: It is the abnormal mechanical loading leading to the excessive expression of CILP in degenerative IVDs, thereby promoting IDD by exerting an inhibitory effect on matrix synthesis.

However, there is a limitation to the present study study; the role of CILP in mechanical stress-mediated regulation of the ECM was not examined, of which the occurrence is implied by the findings of our study but is not directly demonstrated, which 
need further experiments to display. In conclusion, these results have suggested that CILP may be a potential therapeutic target for preventing loss of the ECM in IDD.

\section{Acknowledgements}

Not applicable.

\section{Funding}

The present study was funded by the National Natural Science Foundation of China (grant no. 81071497).

\section{Availability of data and materials}

The analyzed data sets generated during the study are available from the corresponding author on reasonable request.

\section{Authors' contributions}

JH was involved in the conception and design of the study, collection and/or assembly of data, data analysis and interpretation, and manuscript writing. CF was involved in the collection and/or assembly of data, and data analysis and interpretation. KL and JS were involved in the collection and/or assembly of data, and provision of study materials and patients. TC was involved in the conception and design of the study, and the revision of the manuscript. YZ was involved in the conception and design of the study, and provided final approval of the manuscript. YP provided final approval of the manuscript, was involved in the conception and design of the study, and provided financial and administrative support.

\section{Ethics approval and consent to participate}

Human IVDs were obtained with the approval of the Ethical Committee of Xinqiao Hospital (Chongqing, China) and with informed consent, in accordance with the Helsinki Declaration, from individuals undergoing surgery for IDD.

\section{Consent for publication}

Not applicable.

\section{Competing interests}

The authors declare that they have no competing interests.

\section{References}

1. Colombini A,Lombardi G,Corsi MMand Banfi G: Pathophysiology of the human intervertebral disc. Int $\mathrm{J}$ Biochem Cell Biol 40: 837-842, 2008

2. Daly C, Ghosh P, Jenkin G, Oehme D and Goldschlager T: A review of animal models of intervertebral disc degeneration: Pathophysiology, regeneration, and translation to the clinic Biomed Res Int 2016: 1-14, 2016.

3. Seki S, Kawaguchi Y, Chiba K, Mikami Y, Kizawa H, Oya T, Mio F, Mori M, Miyamoto Y, Masuda I, et al: A functional SNP in CILP, encoding cartilage intermediate layer protein, is associated with susceptibility to lumbar disc disease. Nat Genet 37: 607-612, 2005.
4. Yee A, Lam MP, Tam V, Chan WC, Chu IK, Cheah KS, Cheung KM and Chan D: Fibrotic-like changes in degenerate human intervertebral discs revealed by quantitative proteomic analysis. Osteoarthritis Cartilage 24: 503-513, 2016.

5. Wang Z, Kim JH, Higashino K, Kim SS, Wang S, Seki S, Hutton WC and Yoon ST: Cartilage intermediate layer protein (CILP) regulation in intervertebral discs. The effect of age, degeneration, and bone morphogenetic protein-2. Spine (Phila Pa 1976) 37: E203-E208, 2012.

6. Min SK, Nakazato K, Yamamoto Y, Gushiken K, Fujimoto H, Fujishiro H, Kobayakawa Y and Hiranuma K: Cartilage intermediate layer protein gene is associated with lumbar disc degeneration in male, but not female, collegiate athletes. Am J Sports Med 38: 2552-2557, 2010.

7. Min SK, Nakazato K, Ishigami H and Hiranuma K: Cartilage intermediate layer protein and asporin polymorphisms are independent risk factors of lumbar disc degeneration in male collegiate athletes. Cartilage 5: 37-42, 2014.

8. Wang W, Hao J, Zheng S, Xiao X, Wen Y, He A, Guo X and Zhang F: Association between cartilage intermediate layer protein and degeneration of intervertebral disc: A Meta-analysis. Spine (Phila Pa 1976) 41: E1244-E1248, 2016.

9. Mayer JE, Iatridis JC, Chan D, Qureshi SA, Gottesman O and Hecht AC: Genetic polymorphisms associated with intervertebral disc degeneration. Spine J 13: 299-317, 2013.

10. Johnson K, Farley D, Hu SI and Terkeltaub R: One of two chondrocyte-expressed isoforms of cartilage intermediate-layer protein functions as an insulin-like growth factor 1 antagonist. Arthritis Rheum 48: 1302-1314, 2003 .

11. Yao Z, Nakamura H, Masuko-Hongo K, Suzuki-Kurokawa M, Nishioka K and Kato T: Characterisation of cartilage intermediate layer protein (CILP)-induced arthropathy in mice. Ann Rheum Dis 63: 252-258, 2004.

12. Seki S, Tsumaki N, Motomura H, Nogami M, Kawaguchi Y, Hori T, Suzuki K, Yahara Y, Higashimoto M, Oya T, et al: Cartilage intermediate layer protein promotes lumbar disc degeneration. Biophys Res Commun 446: 876-881, 2014.

13. Huang M, Wang HQ, Zhang Q, Yan XD, Hao M and Luo ZJ: Alterations of ADAMTSs and TIMP-3 in human nucleus pulposus cells subjected to compressive load: Implications in the pathogenesis of human intervertebral disc degeneration. J Orthop Res 30: 267-273, 2012.

14. Emanuel KS, Vergroesen PP, Peeters M, Holewijn RM, Kingma I and Smit TH: Poroelastic behaviour of the degenerating human intervertebral disc: A ten-day study in a loaded disc culture system. Eur Cell Mater 29: 330-341, 2015.

15. MacLean JJ, Lee CR, Alini M and Iatridis JC: Anabolic and catabolic mRNA levels of the intervertebral disc vary with the magnitude and frequency of in vivo dynamic compression. J Orthop Res 22: 1193-1200, 2004.

16. Gao X, Zhu Q and Gu W: Prediction of glycosaminoglycan synthesis in intervertebral disc under mechanical loading. J Biomech 49: 2655-2661, 2016.

17. Mori M, Nakajima M, Mikami Y, Seki S, Takigawa M, Kubo T and Ikegawa S: Transcriptional regulation of the cartilage intermediate layer protein (CILP) gene. Biochem Biophys Res Commun 341: 121-127, 2006.

18. Le Maitre CL, Frain J, Millward-Sadler J, Fotheringham AP, Freemont AJ and Hoyland JA: Altered integrin mechanotransduction in human nucleus pulposus cells derived from degenerated discs. Arthritis Rheum 60: 460-469, 2009.

19. Livak KJ and Schmittgen TD: Analysis of relative gene expression data using real-time quantitative PCR and the 2(-Delta Delta C(T)) method. Methods 25: 402-408, 2001.

20. Iorio JA, Jakoi AM and Singla A: Biomechanics of degenerative spinal disorders. Asian Spine J 10: 377-384, 2016.

21. Neidlinger-Wilke C, Würtz K, Urban JP, Börm W, Arand M, Ignatius A, Wilke HJ and Claes LE: Regulation of gene expression in intervertebral disc cells by low and high hydrostatic pressure. Eur Spine J 15 (Suppl 3): S372-S378, 2006.

22. Li P, Gan Y, Wang H, Zhang C, Wang L, Xu Y, Song L, Li S, Li S, Ou Y and Zhou Q: Dynamic compression effects on immature nucleus pulposus: A study using a novel intelligent and mechanically active bioreactor. Int J Med Sci 13: 225-234, 2016.

23. Bleuel J, Zaucke F, Brüggemann GP and Niehoff A: Effects of cyclic tensile strain on chondrocyte metabolism: A systematic review. PLoS One 10: e0119816, 2015. 